\title{
AOR
}

Selected Papers of \#AolR2021:

The 22nd Annual Conference of the

Association of Internet Researchers

Virtual Event / 13-16 Oct 2021

\section{NÜZHUBO AND HER "BROTHERS": STREAMING LIFE AND MEDIATING INTIMACY AT SCALE}

Jingyi Gu

University of Illinois at Urbana-Champaign

\section{Gender and Sexual Dynamics in Live/Life Streaming}

At 11:30 p.m. Chinese local time, I logged onto the Huajiao mobile app and entered the live streaming session hosted by Little Monroe, a 20s-looking woman with heavy makeup and showing a bit of cleavage. She greeted me: "Welcome to my live streaming, brother Gulu." Little Monroe then addressed her over 6,000 viewers in the session: "How's everyone's work today?" Someone responded in the chat box: "I just got off work so I'm exhausted, how about you?" Little Monroe belongs to a group called nüzhubo, which in Chinese means "female live streamers." Many nüzhubos work in a genre I call "live/life streaming," in which they broadcast and discuss their everyday lives to viewers in real-time in exchange for comments and virtual gifts. However, this live streaming genre often finds itself under the scrutiny of a combined state and industry censorship apparatus for creating sexually charged content. Why did Little Monroe assume my gender identity and address me and all other viewers with "brother", a common term of endearment in Chinese? What is the appeal of Little Monroe's session for those 6,000 people who participate in real time?

To understand how seemingly intimate social relationships are formed within nüzhubos and their viewers, as well as mediated through the media technology of live streaming, I conducted 8-months digital ethnography on two Chinese live streaming platform, Inke and Huajiao. The evolving landscape of Chinese live streaming reveals a major gender imbalance, as market research shows that more than $60 \%$ of professional live streamers are women, while in contrast, around $75 \%$ of the active viewership of live streaming consists of men in the 20-40 age group (Business Insider 2016). Live/life streaming as a media genre appeal to their viewers by providing opportunities to have spontaneous interactions with other people based on the exchange of information about their everyday lives. Eventually, gendered and sexual content finds its way into this genre as a result of the live streamers' needs to catch the attention of their viewers. It is often compared to web-camming in Western contexts, regarding that nüzhubos play upon sexual tensions with their predominantly heterosexual male viewers in exchange for money (Cunningham et al., 2019). 


\section{Mediated Intimacy at Scale}

How to understand the relationship between nüzhubos and their viewers is at the center of my inquiry, especially because these most personal mediated encounters happenparadoxically- with huge number of strangers simultaneously in real time. I build on the theoretical framework of "mediated intimacy" to take account of the driving forces behind the formation of this relationship as not only personal and social, but also technical. Concerning the medium through which intimacy is established, media and cultural studies coined the term "mediated intimacy" referring to "personal connections" framed within the "networked public culture" on digital platforms such as Facebook (boyd, 2010; Chambers, 2013; Gregg, 2011) or through mobile devices (Hjorth \& Lim, 2012). Influenced by the feminist and queer critique of neoliberalism, studies on mediated intimacy also underline the labor politics and the commercialization of intimacy (Burke, 2016; Horchschild, 2012; Wang, 2020). To challenge the traditional discourse of intimacy that only refers to love and sex of heteronormative gender and sexuality (Attwood et al., 2017), theorists have expanded the scope of intimacy, considering its non-sexual and non-normative possibilities (Chambers, 2013; Giddens,1992), to denote "a complex sphere of inmost relationships with self and others" in modern subjects' search for "authenticity, meaning, and freedom" (Plummer, 2004). While this body of literature has considered the diverse nature of intimate relationships, the impact of digital technologies, and the gendered aspect of care, there is still need for broadening the theoretical discussions to address how "live" media technologies define and mediate "intimacy at scale," as in the case of Chinese live/life streaming.

\section{Methodology}

To understand how everyday experience of gender, sexuality, and intimacy is mediated at scale within live/life streaming as a digital anthropological site, I will first employ the walkthrough method to directly interact with Huajiao and Inke's interfaces for identifying the gendered and sexual implications in the cultural references and mechanisms of different functions, and the assemblage of various interfaces. Then I follow Hine's (2015) "embedded, embodied, and everyday" approach to investigate how the technological architectures of live streaming platforms afford various user practices towards creating intimate connections. This is done through taking notes on how live streamers use various technological functions, including beauty filters and sound effects, alongside assorted modalities of performance and interaction. I also look for embodiments of emotions in viewers' usage of varying forms of responses including text-based comments and virtual gift purchases.

\section{Preliminary Findings}

Drawing from my participatory observations of live/life streaming sessions, I identify patterns of emotional expression and self-disclosure that lie within these interactions and analyze their alignment with and distinction from those that have been considered within the existing theorization of intimacy. I argue that live/life streaming construct seemingly scalable "mediated intimacy," in the one-versus-many semi-public setting, through nurturing gendered performances and building subtle sexual tension between its participants. Within these sessions that can last for 4-12 hours everyday, the live streamer engage with a crowd of audiences, sometimes a few thousands of people all at the same time through the facilitation of technologies. The conversations happening 
in live/life streaming are infused with disclosures of everyday life and encounters, personal experiences and feelings, positive or negative. The live streamer leads the conversation by performing care, or creating emotional expressions that can be resonated, while keeping track of the comments or virtual gifts from viewers. These ambient gestures toward intimacy are colored with a gendered and sexual undertone, and full of pointers to desires for human connections driven by the state of isolation and exhaustion from everyday hustle. Many nüzhubos are also incentivized to dance around the fine line between the feminine and the overly sexual in their demeanors, to ensure the delivery of intimate feelings but to also stay "appropriate" according to the censorship apparatuses of the state. The live streamers and their viewers are also conscious of the gendered nature of their relationships, and habituated to equate the level of connection to the accumulated commercial value of the virtual gifts that are designed to be the currency for intimacy by the live streaming platforms.

\section{References}

Attwood, F., Hakim, J., \& Winch, A. (2017). Mediated intimacies: Bodies, technologies and relationships. Journal of Gender Studies, 26(3), 249-253.

boyd, d. (2010). Social network sites as networked publics: Affordances, dynamics, and implications. In A networked self (pp. 47-66). Routledge.

Burke, N. B. (2016). Intimate commodities: Intimate labor and the production and circulation of inequality. Sexualities, 19(7), 780-801.

Chambers, D. (2013). Social media and personal relationships online intimacies and networked friendship. Basingstoke: Palgrave Macmillan.

Cunningham, S., Craig, D., \& Lv, J. (2019). China's livestreaming industry: Platforms, politics, and precarity. International Journal of Cultural Studies, 22(6), 719-736.

Giddens, A. (1992). The Transformation of Intimacy: Sexuality, Love and Eroticism in Modern Societies. Stanford University Press.

Gregg, M. (2013). Work's intimacy. John Wiley \& Sons.

Hine, C. (2015). Ethnography for the Internet: Embedded, Embodied and Everyday (1st edition). Routledge.

Hjorth, L., \& Lim, S. S. (2012). Mobile intimacy in an age of affective mobile media. Feminist Media Studies, 12(4), 477-484.

Hochschild, A. R. (2012). The Managed Heart: Commercialization of Human Feeling. University of California Press.

Plummer, K. (2004). Intimate citizenship: Private decisions and public dialogues. Montréal: McGill-Queens University Press. 
Wang, S. (2019). Chinese affective platform economies: Dating, live streaming, and performative labor on Blued: Media, Culture \& Society. 